\title{
Breath Figures under Electrowetting: Electrically Controlled Evolution of Drop Condensation Patterns
}

\author{
Davood Baratian, Ranabir Dey, Harmen Hoek, Dirk van den Ende, and Frieder Mugele \\ Physics of Complex Fluids, MESA+ Institute for Nanotechnology, University of Twente, \\ P.O. Box 217, 7500 AE Enschede, The Netherlands
}

(Received 17 January 2018; published 24 May 2018)

\begin{abstract}
We show that electrowetting (EW) with structured electrodes significantly modifies the distribution of drops condensing onto flat hydrophobic surfaces by aligning the drops and by enhancing coalescence. Numerical calculations demonstrate that drop alignment and coalescence are governed by the drop-sizedependent electrostatic energy landscape that is imposed by the electrode pattern and the applied voltage. Such EW-controlled migration and coalescence of condensate drops significantly alter the statistical characteristics of the ensemble of droplets. The evolution of the drop size distribution displays self-similar characteristics that significantly deviate from classical breath figures on homogeneous surfaces once the electrically induced coalescence cascades set in beyond a certain critical drop size. The resulting reduced surface coverage, coupled with earlier drop shedding under EW, enhances the net heat transfer.
\end{abstract}

DOI: 10.1103/PhysRevLett.120.214502

Dropwise condensation of water vapor is intrinsic to natural phenomena like dew formation [1] and dew or fog harvesting by animals (e.g., Namib Desert beetle) and plants such as Namib Desert plants [2]. Dropwise condensation of vapor is also utilized in various technologies like water-harvesting systems [3], heat exchangers for cooling systems [4], and desalination systems [5]. The efficacy of these technologies depends on the nucleation, coalescence, and growth of the condensate droplets on surfaces and on their subsequent shedding [6,7]. The pattern formed by condensing droplets is classically referred to as a breath figure due to its similarity with the pattern formed by breathing on a cold surface [8-10]. An intriguing feature of breath figures is that the pattern evolution of condensate droplets is self-similar in time, as established by scaling the droplet size distribution [11-15]. To improve the efficiency of the dropwise condensation process, it is essential to control the underlying breath figure characteristics.

In general, dropwise condensation can be controlled by regulating the mobility of the droplets on the surface. Enhanced dropwise condensation has been studied on superhydrophobic nanostructured surfaces [16-19], on superhydrophobic microgrooved and wettability-patterned surfaces [20,21], and on liquid impregnated textured surfaces [22,23]. However, in all the studies so far, droplet mobility is altered passively by tailoring the characteristics of the condensing surface through chemical or topographical patterning. In this Letter, we go beyond such passive approaches and use an alternating (ac) electric field in an electrowetting (EW) configuration with patterned electrodes [24] to actively control the breath figure evolution on otherwise homogeneous hydrophobic surfaces. We demonstrate that EW significantly modifies the distribution of condensate drops using electrical forces and alters the statistical characteristics of the entire ensemble of droplets from those established for classical breath figures with randomly distributed drops. The breath figure evolution under EW is characterized by size-dependent alignment of the condensate drops at the minima in the corresponding electrostatic energy landscapes and enhanced coalescence. A scaling analysis shows that the size distribution of drops is self-similar in time with different characteristics in the initial and final growth phases. The unique transformation in the scaling plot is triggered by EW-induced coalescence cascades beyond a critical drop size. Such EW-mediated alignment and enhanced coalescence increases the average droplet radius, but reduces the surface coverage.

The condensation experiments are performed on a glass plate coated by a hydrophobic dielectric polymer film [Fig. 1(a)]. The glass substrate contains a stripe pattern of transparent interdigitated ITO electrodes [Fig. 1(a)]. The width of both the electrodes and the gaps is $200 \mu \mathrm{m}$. An ac voltage with a frequency of $f=1 \mathrm{kHz}$ and a maximum amplitude of $150 \mathrm{~V} U_{\text {rms }}$ is applied. For the experimental details, see Sec. S1 in the Supplemental Material [25]. For the experiments, a stream of vapor-air mixture at a flow rate of $2 \mathrm{l} / \mathrm{min}$ and a temperature of $36 \pm 1{ }^{\circ} \mathrm{C}$ is passed through a condensation chamber, in which the substrate is kept at a temperature of $25^{\circ} \mathrm{C}$. Condensation experiments are performed both without EW (control) and under EW on identical substrates and under identical experimental conditions. The condensation on the control and EWfunctionalized surfaces is monitored using a high resolution camera. We denote the time instant at which reliably detectable condensate drops of radius $\sim 5 \mu \mathrm{m}$ are detected 

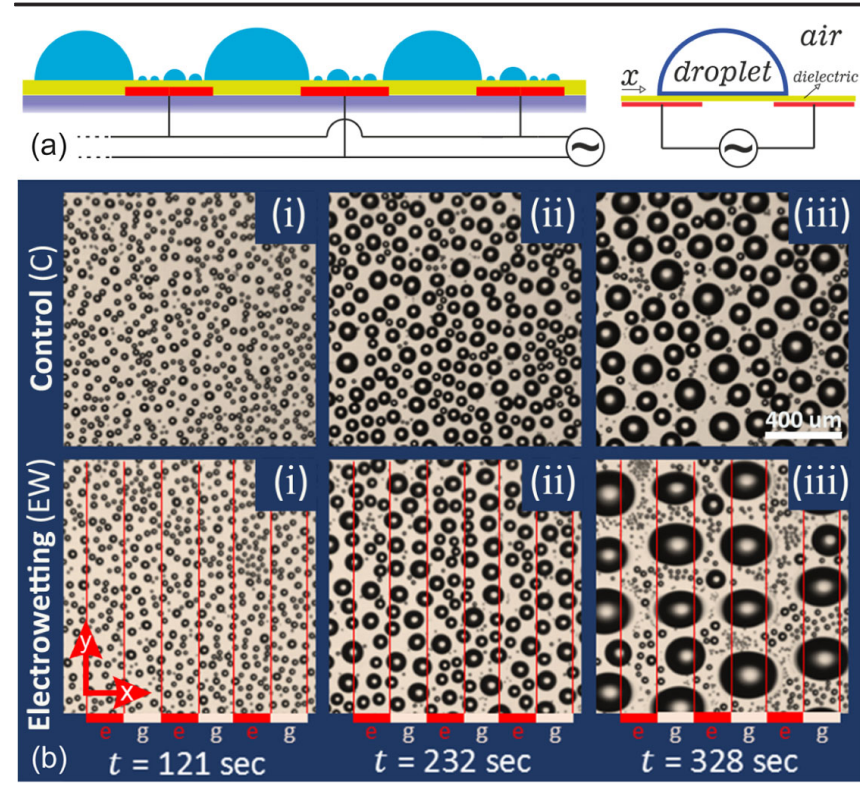

FIG. 1. (a) Schematic of the substrate used for the condensation experiments. Transparent interdigitated ITO electrodes (red) are patterned on the glass substrate (gray), which is then coated with a hydrophobic dielectric polymer film (green). A schematic of a condensate droplet under EW is also shown. (b) Comparison between breath figures without EW [control (C)] [C(i)-C(iii)] and under $\mathrm{EW}\left(U_{\mathrm{rms}}=150 \mathrm{~V} ; f=1 \mathrm{kHz}\right)[\mathrm{EW}(\mathrm{i})-\mathrm{EW}(\mathrm{iii})]$ at different time $(t)$ instants. The electrode-gap (e, g) geometry underneath the dielectric film is indicated by the solid red and white lines. Gravity points from top to bottom, i.e., along the negative $y$ direction.

for the first time as $t=0 \mathrm{~s}$ (for the image analysis procedure see Sec. S8 in [25]).

At the beginning, small drops appear at random locations on both the control and the EW-functionalized surfaces and grow without coalescence [Fig. 1(b), C(i), EW(i)]. As the drops grow and coalesce frequently (see Video S1 in [25]), the drops on the EW-functionalized surface initially align parallel to the electrode edges, displaced towards the gap centers [Fig. 1(b), EW(ii)]. As the droplets grow further and exceed a critical size, sequences of rapid coalescence events (coalescence cascades) create approximately monodisperse drops bridging the gap $\left(w_{g}\right)$ between two adjacent electrodes and aligned along the gap centers [Fig. 1(b), EW(iii); Fig. S2 in [25]]. At the same time, the drops on the control surface remain randomly distributed and display more polydispersity and smaller average sizes [Fig. 1(b), $\mathrm{C}($ iii)]. The transformation in the breath figure under EW [Fig. 1(b), EW(ii)-EW(iii)] occurs within a narrow transition period. Eventually, the gravity-induced drop shedding occurs earlier under EW compared to the control surface (Fig. S3 in [25]).

To quantify the unique distribution of droplets under $\mathrm{EW}$, we project the droplets in the breath figure onto unit cells of width equal to the pitch $(l)$ of the electrode pattern and ranging from one electrode center to the adjacent
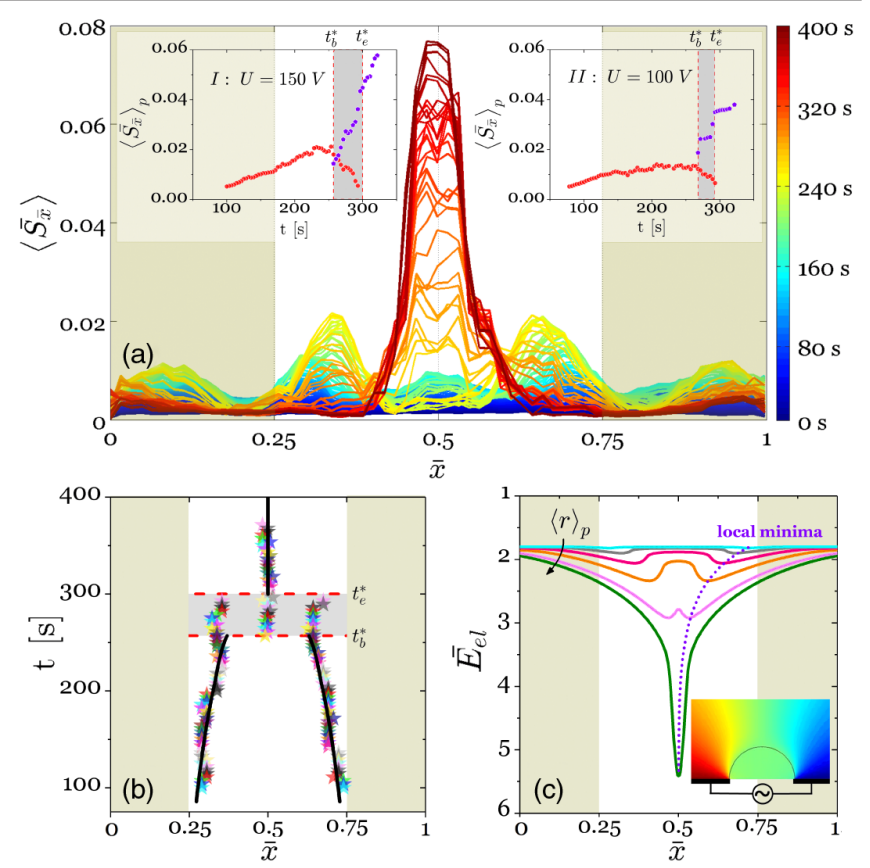

FIG. 2. (a) Color-coded temporal evolution of the average area fraction distribution of drops $\left\langle\bar{S}_{\bar{x}}\right\rangle$ along the nondimensionalized lateral coordinate $(\bar{x}=x / l)$, over one pitch of the electrode pattern under EW $\left(U_{\text {rms }}=150 \mathrm{~V}\right)$. The temporal variations of the peak values $\left\langle\bar{S}_{\bar{x}}\right\rangle_{p}$ in $\left\langle\bar{S}_{\bar{x}}\right\rangle$ for different voltages are shown in insets: (I) $U_{\text {rms }}=150 \mathrm{~V}$, (II) $U_{\text {rms }}=100 \mathrm{~V}$. The temporal variations of the peak locations in $\left\langle\bar{S}_{\bar{x}}\right\rangle$ are shown by star markers in (b). The transition period is shown by the gray area in the insets in (a) and in (b). (c) The nondimensionalized electrostatic energy $\bar{E}_{\mathrm{el}}$ landscapes corresponding to representative values of the area-weighted average radius $\left(\langle r\rangle_{p}=20,40,60,80,100,110 \mu \mathrm{m}\right)$ of the drops constituting the peak(s) in $\left\langle\bar{S}_{\bar{x}}\right\rangle$ before (with two minima) and after (with a single minimum) transition. The evolution in the electrostatic energy minima locations with increasing $\langle r\rangle_{p}$ is shown by the solid black line in (b). The inset shows the electric potential $\varphi$ distribution for a condensate droplet under EW [schematic in Fig. 1(a)].

electrode center. Within a unit cell, we calculate the variation of the droplet area fraction $\left(\bar{S}_{x}\right)$ along the lateral $(x-)$ direction. Here, $\bar{S}_{x}=\sum_{i} A_{i} / A_{\mathrm{uc}}$, where $A_{i}$ is the $i$ th droplet contact area along a line parallel to the electrode edges ( $y$ direction), and $A_{\mathrm{uc}}$ is the unit cell area; $\left\langle\bar{S}_{\bar{x}}\right\rangle$ represents the average over all unit cells in the breath figure, and $\bar{x}=x / l$. The temporal evolution of $\left\langle\bar{S}_{\bar{x}}\right\rangle$ shows the gradual development of two similar peaks from the gap edges [Fig. 2(a)]. The development of these local maxima describes the alignment of the condensate drops on both sides of the gap center [Fig. 1(b), EW(ii)]. In contrast, the corresponding $\left\langle\bar{S}_{\bar{x}}\right\rangle$ distribution on the control surface remains uniform (Fig. S4 in [25]). At any time instant, the peak value $\left\langle\bar{S}_{\bar{x}}\right\rangle_{p}$ describes the maximum droplet coverage. Over time, as the droplets coalesce and grow (see Video S1), $\left\langle\bar{S}_{\bar{x}}\right\rangle_{p}$ gradually increases [red symbols in 
inset (I) in Fig. 2(a)], and concurrently, the locations of the two peaks gradually shift towards the gap center [symbols in Fig. 2(b)]. As the average radius of the drops constituting a peak $\langle r\rangle_{p}$ exceeds a critical value $\langle r\rangle_{p}^{*} \sim 0.3 w_{g}$, the coalescence cascades set in. Consequently, a new peak in $\left\langle\bar{S}_{\bar{x}}\right\rangle$ emerges at the gap center [purple symbols in inset (I) in Fig. 2(a)] while the side peaks start to decay; this marks the beginning $\left(t_{b}^{*}\right)$ of the transition period. Within the transition period, the side peaks disappear and the peak at the gap center survives in the end $\left(t_{e}^{*}\right)$ [Fig. 2(a) and inset (I), and Fig. 2(b)]. This single peak in $\left\langle\bar{S}_{\bar{x}}\right\rangle$ reflects the alignment of the bigger condensate drops at the gap center [Fig. 1(b), EW(iii)]. Beyond $t_{e}^{*}$, these drops grow [Fig. 2(a) and inset (I)] while remaining aligned along the gap center [Fig. 2(b)]. A similar breath figure evolution is observed for other values of the applied voltage, as shown by the $\left\langle\bar{S}_{\bar{x}}\right\rangle_{p}$ evolution at $U_{\mathrm{rms}}=100 \mathrm{~V}$ [inset (II) in Fig. 2(a)].

To understand the evolution of $\left\langle\bar{S}_{\bar{x}}\right\rangle$ under EW, we calculate the electrostatic energy $E_{\text {el }}$ profile over the unit cell for a representative condensate droplet of variable size [schematic in Fig. 1(a)]. For a droplet having radius $r=\langle r\rangle_{p}$, the electric potential $\varphi$ corresponding to an $x$ position is calculated by solving $\nabla \cdot\left[\left(\epsilon_{0} \epsilon-i(\sigma / \omega)\right) \nabla \varphi\right]=0$ using a finite element method [inset in Fig. 2(c)]. Here, $\epsilon_{0}$ is the vacuum permittivity, $\epsilon$ is the material dielectric constant, $\sigma$ is the material electrical conductivity, and $\omega$ is the circular frequency. For the present experimental conditions, the contribution of polarization current is significantly small compared to the conductive contribution. Hence, the system operates in the regime of ac-EW rather than "dielectrowetting" [26] (see Sec. S9 in [25] for numerical computation details). Thereafter, the total electrostatic energy of the system is calculated as $E_{\mathrm{el}}=-\int_{v} \frac{1}{2} \vec{E} \cdot \epsilon_{0} \epsilon \vec{E} d v$, where $\vec{E}=$ $-\nabla \varphi$ is the electric field, and $v$ is the computation domain volume. Subsequently, the $E_{\mathrm{el}}(x)$ landscape (nondimensionalized as $\bar{E}_{\mathrm{el}}=\left|E_{\mathrm{el}}\right| / 4 \pi\langle r\rangle_{p}^{2} \gamma ; \gamma$ is the water-air surface tension) is evaluated as a function of the drop position along the unit cell. Before the onset of coalescence cascade, i.e., for $\langle r\rangle_{p} \lesssim\langle r\rangle_{p}^{*}, \bar{E}_{\text {el }}$ is symmetric about the gap center with minima (electrostatic potential wells) on either side of it [Fig. 2(c)]. The locations of these two potential wells gradually shift towards the gap center with increasing $\langle r\rangle_{p}$ [dotted line in Fig. 2(c)]. The temporal evolution of the peak locations in $\left\langle\bar{S}_{\bar{x}}\right\rangle$ (symbols) closely follows the electrostatic energy minima locations (solid black line) corresponding to increasing $\langle r\rangle_{p}$ [Fig. 2(b)]. The drops formed due to coalescence under EW thus migrate to the electrostatic energy minima corresponding to their sizes, culminating in the alignment of the drops on either side of the gap center. As $\langle r\rangle_{p}$ exceeds $\langle r\rangle_{p}^{*} \sim 0.3 w_{g}$, droplets under the side peaks with $r>\langle r\rangle_{p}^{*}$ migrate towards the corresponding electrostatic energy minima even closer to the gap center. Motion of these droplets initiates collisions between droplets on both sides of the gap center triggering the coalescence cascades (see Sec. S5 in [25]). The resulting drops with diameters comparable to $w_{g}\left(r \gtrsim 0.6 w_{g}\right)$, along with the adjacent electrodes, form two parallel plate capacitors in series, with the dielectric layer as spacers [27]. In this case, $\bar{E}_{\text {el }}$ is symmetric with a single minimum at the gap center [Fig. 2(c)]. Consequently, these approximately monodisperse drops migrate to, and remain aligned along, the gap center [Fig. 2(b)]. Such alignment results in a definite periodicity of the droplet pattern along the $x$ direction, which is determined by the electrode pitch [Fig. 1(b), EW(iii)]. Furthermore, EW-induced coalescence cascades result in a sharp increase of the area-weighted average radius $\left(\langle r\rangle=\sum r^{3} / \sum r^{2}\right)$ of the droplets (Fig. 3). The larger droplets created due to the coalescence cascades increase $\langle r\rangle$ compared to that for the control surface (Fig. 3). Eventually, the gravity-driven drop shedding under ac-EW sets in at a smaller drop size as compared to the control surface (Fig. 3), due to the reduction of effective contact angle hysteresis under ac-EW [28] and the consequential enhancement of gravity-driven drop mobilization [29].

The EW-mediated evolution of the droplet size distribution is also significantly different from that established for classical breath figures. The droplet size $\left(s \sim r^{3}\right)$ distribution under EW is bimodal, exhibiting a powerlaw decay for smaller droplets and a bell-shaped distribution for larger droplets [Fig. 4(a)]. Here, $n_{s}(s, t)$ represents the number of droplets of volume $s$ per unit droplet volume and surface area. The temporal variation of $n_{s}$ is selfsimilar, obeying the scaling relation $[11-13,30]$

$$
n_{s}(t)=s^{-\Theta} f\left(\frac{s}{S}\right)
$$

[Fig. 4(b) and Fig. S7 in [25]]. Here, $S(t) \sim\langle r\rangle^{3}$, and the exponent $\Theta=1+d / D$ depends on the dimensionality of

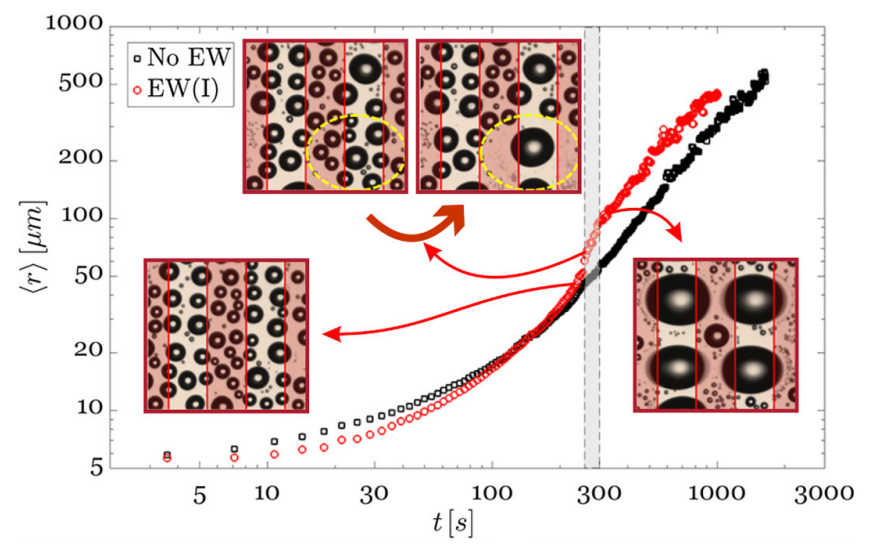

FIG. 3. Evolutions of the area-weighted mean radius $\langle r\rangle$ of the breath figure droplets without EW (control) and under $\mathrm{EW}\left(U_{\mathrm{rms}}=150 \mathrm{~V}\right)$ until the first shedding. The droplet patterns corresponding to different growth regimes of $\langle r\rangle$ under EW are shown as insets. The similar $\langle r\rangle$ variation for $U_{\text {rms }}=100 \mathrm{~V}$ is shown in Fig. S6 in [25]. 
the drops $(D=3)$ and the condensing surface $(d=2)$. For $t<t_{b}^{*}$, the coalescence-dominated evolution of the bellshaped distributions for the larger drops is collapsed by the similarity transformation [Eq. (1)] on considering $\Theta=5 / 3$ [gray shaded region of the scaling plots for $t<t_{b}^{*}$ in Fig. 4(b)]. It must be noted that the smaller drops $(\ln [s / S]<-4)$ follow a different scaling that is dependent on the nucleation and the small scale growth mechanisms $[15,30]$. However, for characterizing the breath figures under EW [Fig. 2(a)], it is sufficient to describe the evolution of the bell-shaped part of the droplet size distribution, since the corresponding droplets align within the gaps. This is substantiated by the fact that the $n_{s}$ estimates corresponding to $s_{p} \sim\left(\langle r\rangle_{p}\right)^{3}$ are close to the peak of the bell-shaped distribution [triangular markers in Fig 4(a)]. Naturally, the evolution of $n_{s}(s, t)$ under EW loses its self-similarity in the transition period due to the EW-induced coalescence cascades [inset in Fig. 4(b)], unlike the droplet size distribution for the control surface, which remains self-similar throughout the coalescence-
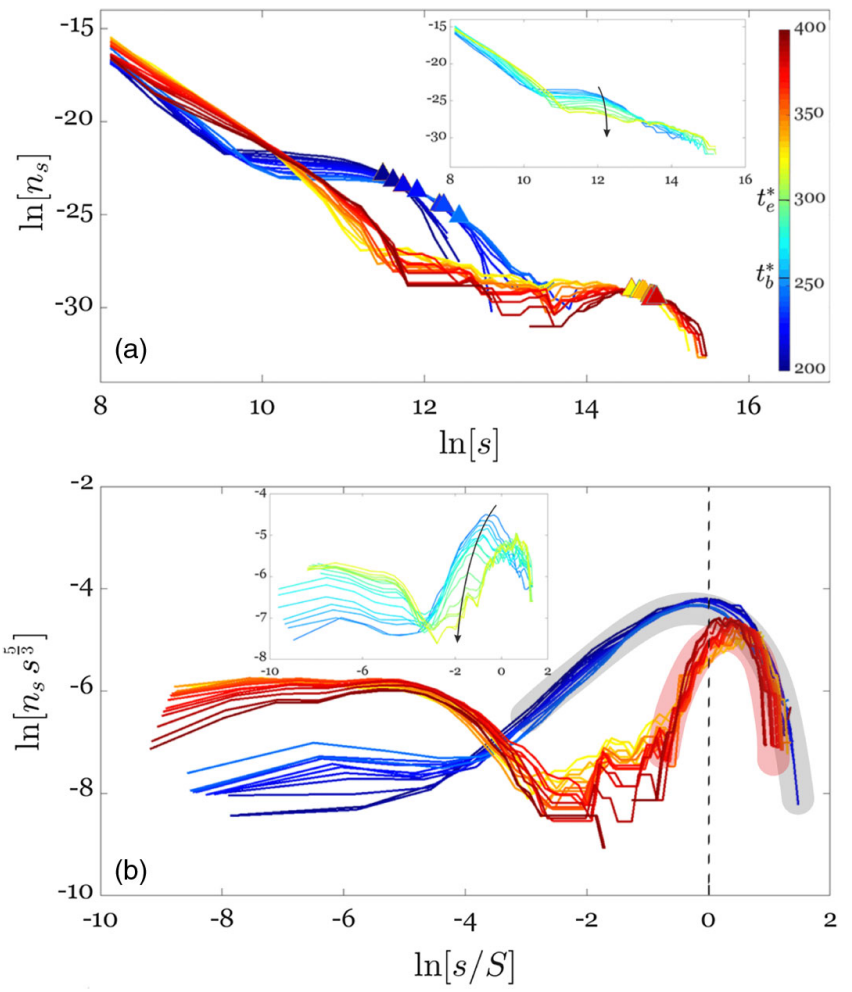

FIG. 4. (a) Self-similar evolution of the droplet size $(s)$ distribution $\left(n_{s}\right)$ in the breath figure under EW $\left(U_{\mathrm{rms}}=150 \mathrm{~V}\right)$ before and after the transition period. The time evolution is color coded with times before and after transition, colored in shades of blue and red, respectively. The triangular markers show the $n_{s}$ estimates for average drop sizes constituting the peaks in $\left\langle\bar{S}_{\bar{x}}\right\rangle$ [Fig. 2(a)]. (b) Corresponding scaling plots of $n_{s}$ obtained using Eq. (1) before and after the transition. The $n_{s}$ evolution within the transition period, and the corresponding scaling plots, are shown in the insets in (a) and (b), respectively. dominated growth regime [Fig. S7 in [25]]. However, for $t \gtrsim t_{e}^{*}$, the evolution of $n_{s}$ for the larger droplets again exhibits self-similarity, albeit with a significantly different functional form compared to that for $t<t_{b}^{*}$ [Fig. 4(b)] and for the control surface [Fig. S7 in [25]]. The almost uniform sizes of the large droplets created due to the coalescence cascades manifest in the narrower bell-shaped distribution for $t \gtrsim t_{e}^{*}$ [compare the blue versus red scaling plots in Fig. 4(b)]. Furthermore, due to the large separation of sizes between the dominant monodisperse droplets and the small background droplets [Fig. 1(b), EW(iii)], the corresponding bell-shaped distributions are no longer peaked at $S$, contrary to that for $t<t_{b}^{*}$ and for the control surface [Fig. 4(b); Fig. S7 in [25]]. Interestingly, the alignment of the growing monodisperse drops at the electrostatic energy minima, which always remains at the gap center, makes the corresponding size distribution analogous to that for condensation on a line $(d=1)$ [12]. Such evolution of the droplet size distribution is unique to the EW-induced anisotropy in the system. Furthermore, the significant reduction in $n_{s}$ for the relatively larger droplets, due to the coalescence cascades [Fig. 4(b)], results in enhanced "release" of substrate surface area. Consequently, we observe $\sim 10 \%-12 \%$ reduction in the steady state surface coverage $\left(\Gamma=\sum \pi r^{2} / A_{\mathrm{FOV}}\right.$, where $A_{\mathrm{FOV}}$ is the area of the field of view) under EW, with respect to the control surface (Fig. 5). The reduced $\Gamma$ coupled with the enhanced gravitydriven shedding of condensate drops, due to the ac-EWinduced increased droplet growth and reduced hysteresis, results in enhanced heat transfer (inset in Fig. 5). Preliminary heat transfer measurements show a striking 50\%-60\% increase in net heat flux for dropwise condensation under EW (inset in Fig. 5). It must be noted that thinner inorganic dielectric layers (e.g., $\mathrm{SiO}_{2}$ or $\mathrm{Al}_{2} \mathrm{O}_{3}$ ) are available for $\mathrm{EW}[31,32]$ to significantly minimize the

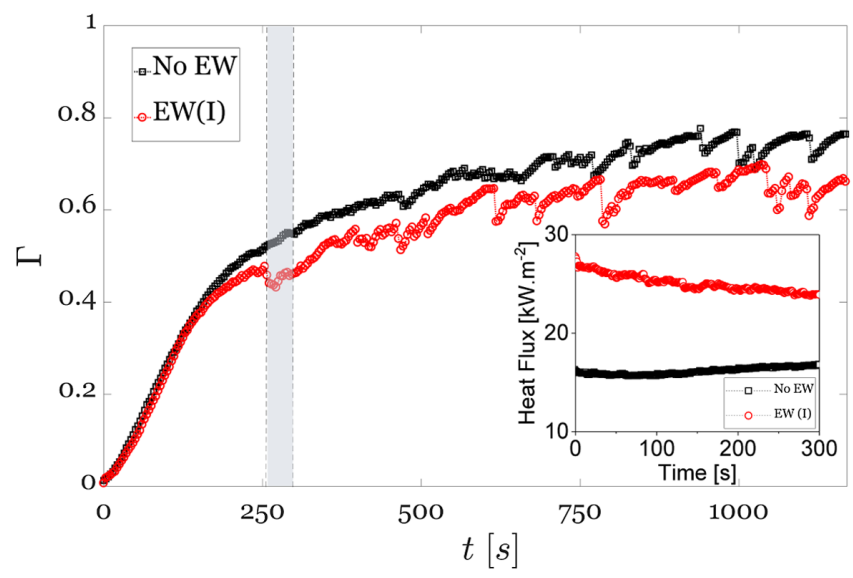

FIG. 5. Evolutions of the breath figure surface coverage $(\Gamma)$ without EW (control) and under $\mathrm{EW}\left(U_{\text {rms }}=150 \mathrm{~V}\right)$. Inset shows the enhanced heat flux under EW. Heat transfer data are recorded in a separate setup; see Sec. S10 in [25] for details. 
thermal resistance compared to the presently used polymer layers.

In summary, we have shown that the electrostatic energy landscape under ac-EW induces migration and coalescence of condensate droplets, leading to unique breath figure characteristics. The coalescing drops align at the corresponding electrostatic energy minima, instead of staying restricted to the center of mass of the parent drops as in classical breath figures. The resulting periodicity of the droplet pattern under EW can be further controlled by simply tuning the electrode geometry. We hope that this study will trigger a general theoretical analysis of drop condensation patterns in arbitrary energy landscapes. We have also shown that the modification of the breath figure characteristics under EW leads to enhanced heat transfer. We anticipate that these effects will be very useful for optimizing applications involving dropwise condensation, like heat exchangers and breath figure templated selfassembly [33].

We sincerely thank Kripa Varanasi for allowing us to use the heat transfer measurement setup in his lab and Karim Khalil for help during the heat transfer measurements. We thank Arjen Pit for assistance with the numerical calculations, and Daniel Wijnperle and B. Robert for their help with the preparation of the condensation substrates. We acknowledge financial support by the Dutch Technology Foundation STW, which is part of the Netherlands Organization for Scientific Research (NWO), and the VICI program (Grant No. 11380).

D. B. and R. D. contributed equally to this work.

*f.mugele@utwente.nl

[1] D. Beysens, Atmos. Res. 39, 215 (1995).

[2] F. Malik, R. Clement, D. Gethin, W. Krawszik, and A. Parker, Bioinspiration Biomimetics 9, 031002 (2014).

[3] D. Milani, A. Abbas, A. Vassallo, M. Chiesa, and D. Al Bakri, Chem. Eng. Sci. 66, 2491 (2011).

[4] M.-H. Kim and C. W. Bullard, Int. J. Refrig. 25, 924 (2002).

[5] A. D. Khawaji, I. K. Kutubkhanah, and J.-M. Wie, Desalination 221, 47 (2008).

[6] D. Beysens, C.R. Phys. 7, 1082 (2006).

[7] J. Rose, Int. J. Heat Mass Transfer 10, 755 (1967).

[8] D. Beysens and C. M. Knobler, Phys. Rev. Lett. 57, 1433 (1986).

[9] J. L. Viovy, D. Beysens, and C. M. Knobler, Phys. Rev. A 37, 4965 (1988).

[10] D. Fritter, C. M. Knobler, and D. A. Beysens, Phys. Rev. A 43, 2858 (1991).

[11] F. Family and P. Meakin, Phys. Rev. Lett. 61, 428 (1988).

[12] F. Family and P. Meakin, Phys. Rev. A 40, 3836 (1989).

[13] P. Meakin, Rep. Prog. Phys. 55, 157 (1992).
[14] R. D. Narhe, M. D. Khandkar, K. P. Adhi, A. V. Limaye, S. R. Sainkar, and S. B. Ogale, Phys. Rev. Lett. 86, 1570 (2001).

[15] J. Blaschke, T. Lapp, B. Hof, and J. Vollmer, Phys. Rev. Lett. 109, 068701 (2012).

[16] J. B. Boreyko and C.-H. Chen, Phys. Rev. Lett. 103, 184501 (2009).

[17] N. Miljkovic, R. Enright, and E. N. Wang, ACS Nano 6, 1776 (2012).

[18] N. Miljkovic, R. Enright, Y. Nam, K. Lopez, N. Dou, J. Sack, and E. N. Wang, Nano Lett. 13, 179 (2013).

[19] N. Miljkovic, D. J. Preston, R. Enright, and E. N. Wang, ACS Nano 7, 11043 (2013).

[20] R. D. Narhe and D. A. Beysens, Phys. Rev. Lett. 93, 076103 (2004).

[21] A. Ghosh, S. Beaini, B. J. Zhang, R. Ganguly, and C. M. Megaridis, Langmuir 30, 13103 (2014).

[22] S. Anand, A. T. Paxson, R. Dhiman, J. D. Smith, and K. K. Varanasi, ACS Nano 6, 10122 (2012).

[23] H. Tsuchiya, M. Tenjimbayashi, T. Moriya, R. Yoshikawa, K. Sasaki, R. Togasawa, T. Yamazaki, K. Manabe, and S. Shiratori, Langmuir 33, 8950 (2017).

[24] F. Mugele and J.-C. Baret, J. Phys. Condens. Matter 17, R705 (2005).

[25] See Supplemental Material at http://link.aps.org/ supplemental/10.1103/PhysRevLett.120.214502 for Video $\mathrm{S} 1$ of dropwise condensation with and without EW, details of the experimental setup and methodology, image analysis procedure, numerical computation methodology, brief overview of the independent heat transfer measurement experiments, examples and explanation for $\mathrm{EW}$-induced coalescence cascades, proof of early shedding of drops under EW, evolution of the area-weighted mean radius of the breath figure pattern for different values of the applied voltage, and average droplet area fraction distribution and evolution of droplet size distribution without EW.

[26] G. McHale, C. V. Brown, M. I. Newton, G. G. Wells, and N. Sampara, Phys. Rev. Lett. 107, 186101 (2011).

[27] D. Mannetje, S. Ghosh, R. Lagraauw, S. Otten, A. Pit, C. Berendsen, J. Zeegers, D. Van Den Ende, and F. Mugele, Nat. Commun. 5, 3559 (2014).

[28] F. Li and F. Mugele, Appl. Phys. Lett. 92, 244108 (2008).

[29] D. t Mannetje, C. Murade, D. Van Den Ende, and F. Mugele, Appl. Phys. Lett. 98, 014102 (2011).

[30] G. I. Barenblatt, Scaling, Self-Similarity, and Intermediate Asymptotics: Dimensional Analysis and Intermediate Asymptotics (Cambridge University Press, Cambridge, England, 1996), Vol. 14.

[31] S. Berry, J. Kedzierski, and B. Abedian, J. Colloid Interface Sci. 303, 517 (2006).

[32] H. Yang, S. Hong, B. Koo, D. Lee, and Y.-B. Kim, Nano Energy 31, 450 (2017).

[33] M. Srinivasarao, J. O. Park, V. Sharma, L. Song, M. S. Barrow, and P. R. Williams, Evaporative Self-Assembly of Ordered Complex Structures (World Scientific, Singapore, 2012), Chap. 6. 